\title{
The First Appearance of Persistent Dementia and Psychosis after a Generalized Sepsis
}

\author{
C Lazzari*, T Rajanna, A Nusair
}

Department of Psychiatry, Fieldhead Hospital, South-West Yorkshire Partnership NHS Foundation Trust, United Kingdom

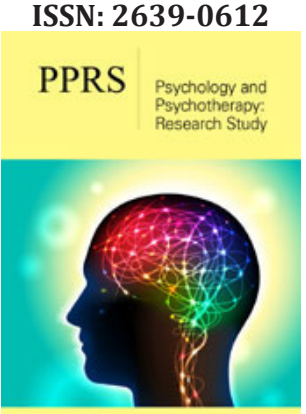

*Corresponding author: Department of Psychiatry, Fieldhead Hospital, South-West Yorkshire Partnership NHS Foundation Trust, United Kingdom

Submission: 眥 March 11, 2019

Published: 眥March 25, 2019

Volume 2 - Issue 3

How to cite this article: C Lazzari, $\mathrm{T}$ Rajanna, A Nusair. The First Appearance of Persistent Dementia and Psychosis after a Generalized Sepsis. Psychol Psychother Res Stud . 2(3).PPRS.000536.2019. DOI: 10.31031/PPRS.2019.02.000536

Copyright@ C Lazzari, This article is distributed under the terms of the Creative Commons Attribution 4.0 International License, which permits unrestricted use and redistribution provided that the original author and source are credited.

\section{Short Communication}

Research suggests that neuroinflammation of the brain has a role in dementia by activating glial cells which then release proinflammatory agents and lipopolysaccharide (LPS) responsible for cognitive deficits [1-3]. Other authors suggest that there is no clear evidence if neuroinflammation after generalized infection is a cause, agent, or consequence of Alzheimer's Disease (AD) with bacterial LPS acting on neurons and microglia [4,5]. The current case study refers to a 64-year old lady who developed symptoms of dementia and psychosis a month after she was operated for a duodenal perforation resulting in pneumoperitoneum and generalized bacterial sepsis. Neuropsychiatric and clinical assessment gathered the required data. Past psychiatric history was negative, and former cognitive deficits were non-existent before the systemic inflammation. At assessment, brain Magnetic Resonance Image presented mild to moderate supratentorial small vessel disease and mild cortical and subcortical atrophy. Addenbrooke's ACE-III total score was 74/100: Attention 18/18, Memory 18/26, Fluency 5/14, Language 20/26 and visuospatial 13/16. The cognitive impairment persisted also after the underlying depression was stabilized. The patient became doubly incontinent and started misplacing objects in her kitchen, confusing the use of familiar objects, and having difficulties in word finding. The Bristol Activity of Daily Living was 10/60. The comprehensive neurocognitive assessment resulted in good episodic memory, poor attention, misplacing objects, social phobia, and subjective memory problems with the inability to complete routine tasks. The neuropsychiatric presentation included believing that her food was poisoned, that one of her daughters was a fake, deeming that people on the road (outside her house) were making derogatory comments about her and passing her intrusive thoughts, and thought block. Passivity experiences consisted in thinking that passers-by were able to interfere with her mind or command her to harm herself. She also had auditory-commanding hallucinations to harm herself, believing deriving from people passing by her house. Other auditory hallucinations included hearing people walking on the stair of her house, hearing someone knocking on the door of her bedroom when no one was there, and tactile hallucinations as if someone was tapping on her shoulders when she was alone in her room. We posed the diagnosis of AD and psychosis. After several months, the presentation remains unchanged. The differential diagnoses (Box 1) utilizing ICD-10 (WHO, International Classification of Diseases) included a severe depressive episode with psychotic symptoms (F32.3), organic depressive disorder (F 06.32), vascular dementia (F01.9), and late-onset schizophrenia (F20.9). All these presentations might have been triggered or caused by brain inflammation targeting specific microglia or brain microcirculation hence resulting in permanent damage leading to the onset of dementia and psychosis. Therefore, the authors of the current research speculate that in the case described, systemic inflammation enduringly unlocked dementia, cognitive deficits, and psychosis [6]. The mediator of the unlocking process could be a hypothetical Alzheimer Triggering Factor linked to the microglia activated by LPS which causes a cascade of processes leading to irreversible dementia. 


\section{BOX1}

\section{DIFFERENTIAL DIAGNOSES}

F32.3 Severe depressive episode with psychotic symptoms: Justification: the nature of auditory hallucination and delusional beliefs about people and reality are mood congruent and in line with depressive symptoms. Initially, auditory hallucinations were derogatory and commanding her to take her life. They remain derogatory.

F06.32 Organic depressive disorder: Justification: The complex and articulate presentation of her symptoms occurred 'after' the sepsis and major intestinal operation. There is no record of previous mental health problems. The hypothesis is of psychosis after major septicemia.

F01.9 Vascular dementia, unspecified: Justification: There are some major cognitive problems in verbal fluency, work finding and expressive aphasia. There has been misplacing of objects in the kitchen. Memory appears not being altered. There is no time, place, person disorientation. The mood is affected by psychotic symptoms.

F20.9 Late-onset schizophrenia, unspecified: Justification: There are cases of late-onset schizophrenia. The primary septicemia might have triggered a dormant psychosis which, now, is not responding to current antipsychotic medication and that is currently assuming more paranoid ideation.

\section{References}

1. Bevan-Jones WR, Surendranathan A, Passamonti L, Patricia Vázquez Rodríguez P, Arnold R, et al. (2017) Neuroimaging of Inflammation in Memory and Related Other Disorders (NIMROD) study protocol: a deep phenotyping cohort study of the role of brain inflammation in dementia, depression and other neurological illnesses. BMJ Open 7(1): e013187.

2. Reale M, Brenner T, Greig NH, Inestrosa N, Paleacu D (2010) Neuroinflammation, AD, and Dementia. Int J Alzheimers Dis 2010: 1-2.

3. Cunnigham C, Campion S, Lunnon K, Murray CL, Woods JFC, et al. (2009) Systemic inflammation induces acute behavioural and cognitive changes and accelerates neurodegenerative disease. Biol Psychiatry 65(4): 304312 .

4. Heneka MT, Carson MJ, El Khoury J, Landreth GE, Brosseron F, et al. (2015) Neuroinflammation in Alzheimer's diseases. Lancet Neurol 14(4): 388-405.

5. Enciu AM, Popescu BO (2013) Is there a causal link between inflammation and dementia? Biomed Res Int 2013: 316495.

6. Lazzari C, Rajanna T, Nusair A, Lyall J (2019) Post-infection dementia and psychosis. Proceedings of the $27^{\text {th }}$ Congress of European Psychiatric Association, Warsaw, Poland. 